\title{
Particle size distribution in FeAg granular alloy
}

\author{
J.M. Soares ${ }^{\text {a }}$, J.H. de Araújo ${ }^{\text {b }}$, F.A.O. Cabral ${ }^{\text {b,c,* }}$, F.L.A. Machado ${ }^{\text {c }}$, T. Dumelow ${ }^{\text {a }}$, M.M. Xavier Jr. ${ }^{\text {d }}$, J.M. Sasaki ${ }^{\text {e }}$ \\ a Departamento de Física, UERN, 59633-010 Mossoró, RN, Brazil \\ b Departamento de Física Teórica e Experimental, UFRN, 59072-970 Natal, Brazil \\ ${ }^{\mathrm{c}}$ Departamento de Física, UFPE, Recife, PE 50670-901, Brazil \\ d Departamento de Física, UFERSA, 59625-900 Mossoró, RN, Brazil \\ e Departamento de Física, UFC, 60455-970 Fortaleza, CE, Brazil
}

\section{A R T I C L E I N F O}

Article history:

Available online 28 August 2008

\section{PACS:}

75.20.En

$75.50 . \mathrm{Bb}$

75.50.Tt

Keyword:

Magnetic properties

\begin{abstract}
A B S T R A C T
A combination of Langevin and hyperbolic tangent functions weighted by a log-normal particle size distributions were used to fit the magnetization curves of $\mathrm{Fe}_{10} \mathrm{Ag}_{90}$ granular alloys, produced by a sol-gel process under different chemical conditions. The fitting technique allows one to get both the superparamagnetic and the blocked particle size distributions. The average size of the Fe particles obtained from the fit were in good agreement with those obtained by X-ray diffraction. The correlation between the coercive field and the particle distribution was also investigated. Zero-field cooling dc magnetic susceptibility measurements were fitted using a similar technique to that used for fitting the magnetization curves. The saturation magnetization and mean Fe particle size values obtained from the two fittings were in good agreement.
\end{abstract}

(c) 2008 Published by Elsevier B.V.

\section{Introduction}

Granular magnetic materials composed of magnetic nanoparticles embedded in a non-magnetic matrix show interesting physical properties such as superparamagnetism, giant magnetoresistance and giant magnetoimpedance [1-3]. Moreover, they have important technological applications in magnetic recording, in optical devices and in sensors [4]. Such materials have been produced as films by sputtering, as ribbons by melt-spinning, and in power form by sol-gel methods [5,6] and by mechanical alloying [7]. The samples produced in these processes can present different particle size distributions.

The shape of both the magnetic susceptibility and the magnetization curves for powder samples are strongly dependent on the sample particle size distribution. Finding the correct distribution is therefore necessary to help the interpretation of some magnetic properties [8-12]. For some materials, the magnetization curves have been fitted with the aid of a log-normal (LN) particle size distribution. In these cases, a distribution of Langevin functions [8], weighted by the LN distribution, is enough to fit the data, indicating a superparamagnetic (SPM) behavior. In other cases, when one has SPM and blocked (BL) particles, the data have been fitted using a combination of weighted Langevin and hyperbolic tangent func-

\footnotetext{
* Corresponding author. Address: Departamento de Física Teórica e Experimental, UFRN, 59072-970 Natal, Brazil. Tel./fax: +55 (81)21268450.

E-mail address: fcabral@ufrnet.br (F.A.O. Cabral).
}

tions [10]. In this work, we analyze magnetization curves using a log-normal distribution in which the particles fall into two distinct regimes: (1) up to a certain limiting diameter are the SPM particles and (2) above it are the BL particles. Langevin and hyberbolic tangent functions weighted by the log-normal distribution are used to fit the experimental magnetization curves yielding parameters that are in good agreement with the values obtained using other techniques.

\section{Experimental}

$\mathrm{Fe}_{10} \mathrm{Ag}_{90}$ granular alloys were produced by a sol-gel method. The start solution was prepared from an aqueous solution of nitrates with five nitric acid concentrations $\left(V_{\mathrm{ac}}\right): 0.25,0.50,0.75$, 1.00 and $1.25 \mathrm{ml}$ (samples $\mathrm{A}, \mathrm{B}, \mathrm{C}, \mathrm{D}$, and $\mathrm{E}$, respectively) in $300 \mathrm{ml}$ of mixture, as detailed in Ref. [13]. This procedure yielded particles with different diameters. The powder obtained was reduced in an hydrogen atmosphere for $45 \mathrm{~min}$ at a temperature of $400{ }^{\circ} \mathrm{C}$. The crystalline structure of the sample was investigated by X-ray diffraction using a Rigaku diffractometer and Mo K $\alpha$ radiation. The samples were also analyzed by transmission electronic microscopy (TEM). Dc magnetization was measured using a vibrating sample magnetometer in the temperature range $300-800 \mathrm{~K}$ and fields of up to $1 \mathrm{~T}$. Zero-field-cooled (ZFC) dc magnetic susceptibility was measured in a magnetic field of 100 Oe whilst heating the sample from room temperature up to $730 \mathrm{~K}$. The field-cooled (FC) dc magnetic susceptibility was measured during the cooling 
of the sample. Mössbauer spectra were obtained using a constant acceleration spectrometer with a ${ }^{57}$ Co source in a rhodium matrix.

\section{Results}

Fig. 1 shows the X-ray diffractometry patterns of $\mathrm{Fe}_{10} \mathrm{Ag}_{90}$ granular alloy reduced at temperature of $400^{\circ} \mathrm{C}$. It is shows that the iron in the $\mathrm{Fe}_{10} \mathrm{Ag}_{90}$ is b.c.c. structure and the silver is f.c.c. structure; there are also some low intensity reflections from $\mathrm{Fe}_{3} \mathrm{O}_{4}$. The inset of Fig. 1 shows details of the X-ray spectra around the $\mathrm{Ag}(222)$ and $\mathrm{Fe}(211)$ Bragg peaks. The solid lines were fitted using two Pseudo-Voigt functions. Applying Scherrer's formula, we obtain the average Fe particle sizes $D_{\mathrm{m}}$ from the (211) linewidth for the nitric acid concentrations $V_{\mathrm{ac}}$. It was observed that $D_{\mathrm{m}}$ increases with $V_{\text {ac }}$ up to $0.75 \mathrm{~mL}$ decreasing after that. Fig. 2 shows the TEM micrographs for the sample A. The image shows the morphology of both the Fe and $\mathrm{Ag}$ particles, and that the particle size distribution is somewhat larger.

Fig. 3 shows the room temperature magnetic hysteresis curve. The coercive field for this sample is 434 Oe. This is a much higher value than that obtained for bulk iron, indicating the presence of sin-

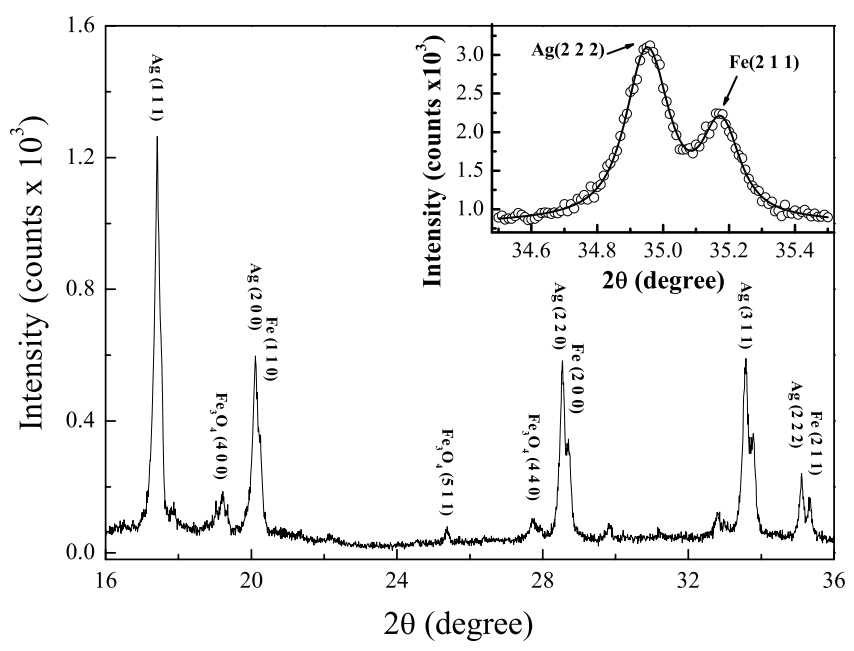

Fig. 1. X-ray diffractogram of sample $C$. The inset shows $\operatorname{Ag}(222)$ and $\mathrm{Fe}(211)$ $\mathrm{X}$-ray peaks for this sample. The solid lines are fitted using two Pseudo-Voigt functions.

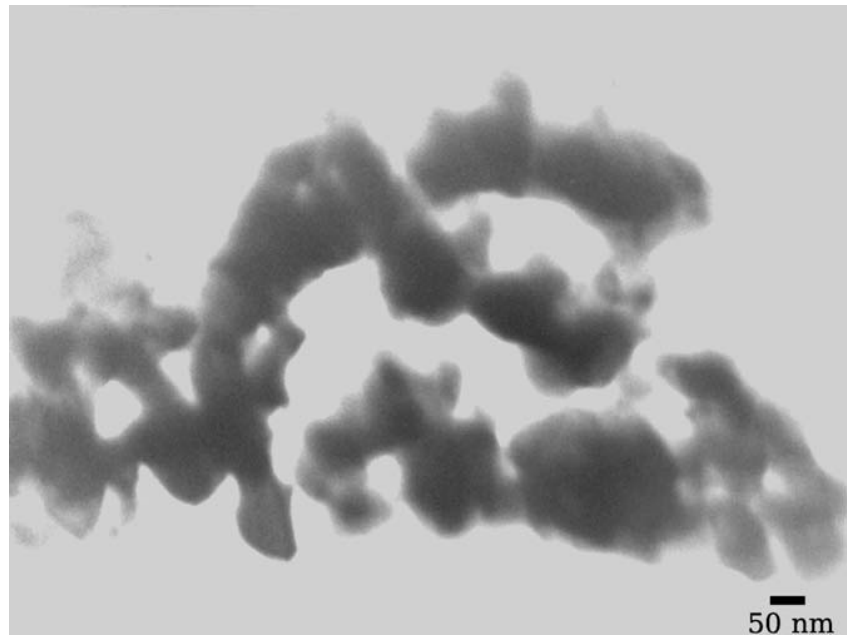

Fig. 2. TEM image for the sample A.

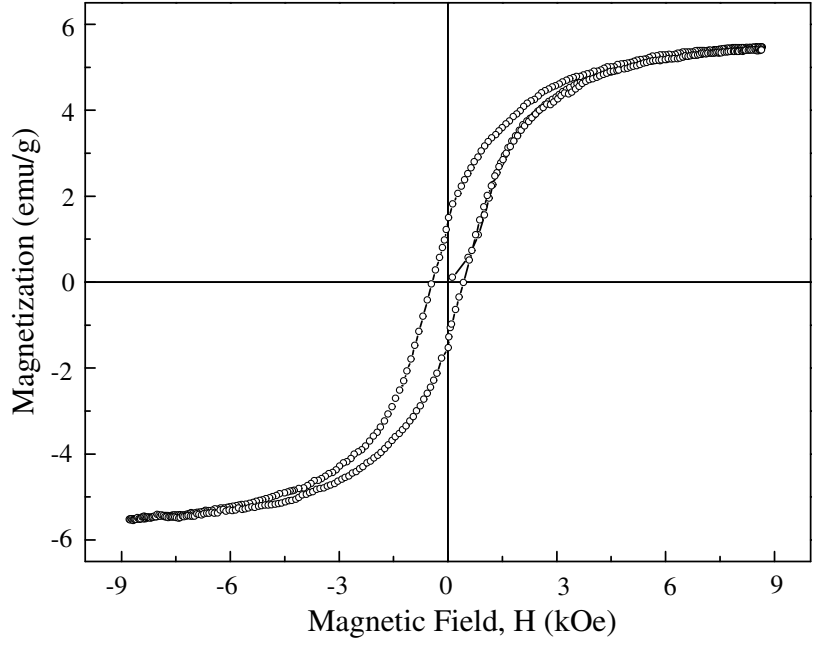

Fig. 3. Room temperature hysteresis curve for sample C.

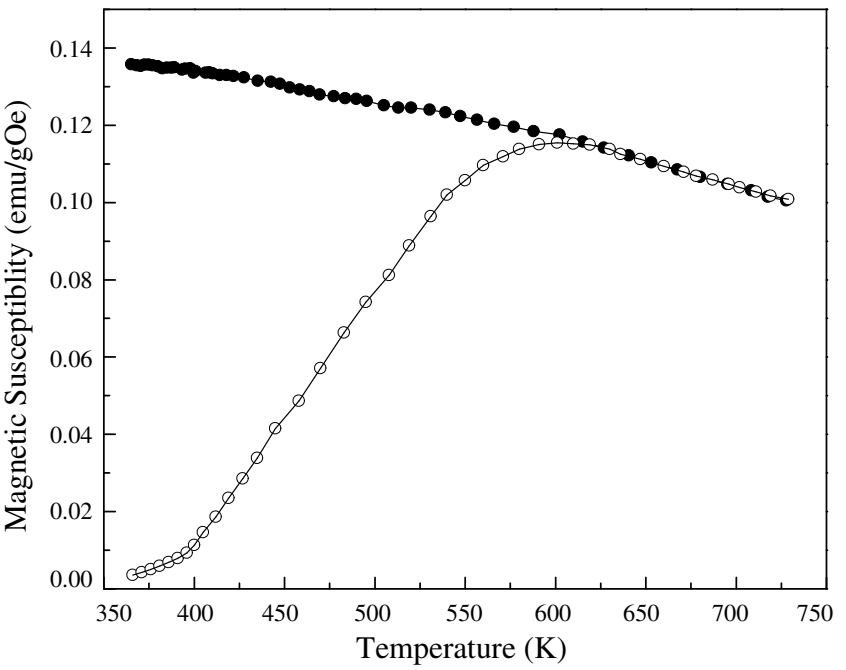

Fig. 4. ZFC (open circles) and FC (closed circles) dc magnetic susceptibility curves for sample $A$ and $H=100$ Oe.

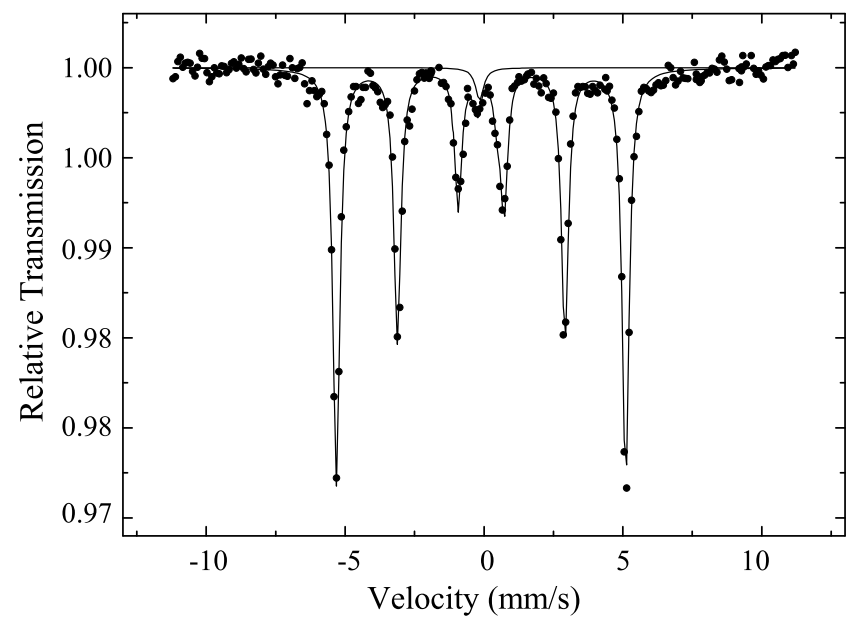

Fig. 5. Mössbauer spectrum showing singlet and sextet subspectra derived from the overall fit.

gle-domain particles. The ZFC and FC magnetic susceptibility curves are shown in Fig. 4, indicating a system of single-domain particles that do not orient themselves spontaneously in the absence of an 
external field, displaying spin glass behavior. The irreversibility in the susceptibility is associated with a transition from a blocked state to a superparamagnetic state. The transition is broad in temperature going from below room temperature up to $600 \mathrm{~K}$. The ZFC and FC curves confirm the presence of both BL and SPM particles at room temperature.

Fig. 5 shows the Mössbauer spectrum for the sample B. One can observe that the spectrum consists of two components: a singlet, characteristic of SPM $\alpha$-Fe particles, and a sextet corresponding to the blocked particles. It is interesting to note that SPM particles are seen even though the measurement time window for the Mössbauer effect is of the order of $10^{-8} \mathrm{~s}$.

\section{Discussion}

The set of experimental results shows that the magnetization of the $\mathrm{Fe}_{10} \mathrm{Ag}_{90}$ samples originates from two contributions: one due to the SPM particles and the other due to the BL particles. Thus, it is reasonable to assume that the log-normal particle size distribution can be divided into two regimes. For a diameter $D<D_{1}$, where $D_{1}$ is a limiting diameter, the particles are SPM, whereas for $D>D_{1}$ they are BL. SPM particles can be well described by Langevin functions while BL particles are described by hyberbolic tangent functions. Therefore, the modified log-normal distribution will weight the Langevin functions from a minimum particle size up to $D_{1}$ and the hyperbolic tangent functions from $D_{1}$ up a maximum particle size. Using this assumption, the magnetization curve can be described by

$M(H)=M_{\mathrm{SPM}} \int_{V_{\min }}^{V_{1}} L\left(\frac{\mu_{\mathrm{SPM}} H}{k_{\mathrm{B}} T}\right) f(V) \mathrm{d} V+M_{\mathrm{BL}} \int_{V_{\mathrm{l}}}^{V_{\max }} \tanh \left(\frac{\mu_{\mathrm{BL}} H}{k_{\mathrm{B}} T}\right) f(V) \mathrm{d} V$,

where $L\left(\frac{\mu H}{k_{\mathrm{B}} T}\right)$ is the Langevin function, $k_{\mathrm{B}}$ is the Boltzman constant, $T$ is the temperature, $\mu_{\mathrm{SPM}}$ and $\mu_{\mathrm{BL}}$ are the magnetic moments of the SPM and BL particles, respectively, $M_{\mathrm{SPM}}$ and $M_{\mathrm{BL}}$ are the magnetizations due to the two particle types, $V=\frac{\pi}{6} D^{3}$ is the volume of the particles assuming that they are spherical, and $f(V)$ is the log-normal distribution function. For spherical particles, the diameter distribution function $f(D)$ can be written as:

$f(D)=\frac{1}{\sqrt{2 \pi \ln \sigma}} \exp \left\{-\frac{\left(\ln D-\ln D_{0}\right)^{2}}{2 \ln ^{2} \sigma}\right\}$,

where $\sigma$ and $D_{0}$ are the width of the distribution and the statistical mean, respectively. The mean diameter of the particle $D_{\mathrm{m}}$ can be calculated from the equation,

$D_{\mathrm{m}}=\frac{\int_{D_{\min }}^{D_{\max }} f(D) D \mathrm{~d} D}{\int_{D_{\min }}^{D_{\max }} f(D) \mathrm{d} D}$

where $D_{\min }$ and $D_{\max }$ are the lower and the upper limit of the distribution, respectively. A Fortran routine was written to obtain a best fit of Eq. (1) to the magnetization data using a least-squares procedure [14]. The integrals were numerically calculated by Gaussian quadratures. Fig. 6(a) shows the magnetization data and the best fit result while Fig. 6(b) shows the particle size distributions obtained using the parameters yielded by the fit. One can see that the distribution width is fairly narrow with $\sigma$ approximately equal to one and particle sizes ranging from 15 to $30 \mathrm{~nm}$. However, when the nitric acid volume is $0.75 \mathrm{ml}$, the distribution width is wider, with $\sigma=1.67$, and particle sizes varying from 5 to $90 \mathrm{~nm}$. When the acid volume is increased further the distribution width becomes narrower, with $\sigma=1.24$. Therefore, the nitric acid concentration in the start solution directly affects the Fe particle size distribution.

The overall limiting diameter obtained was $D_{1}=18 \mathrm{~nm}$, with a maximum deviation of $3 \%$. This $D_{1}$ value is very near to that calcu-
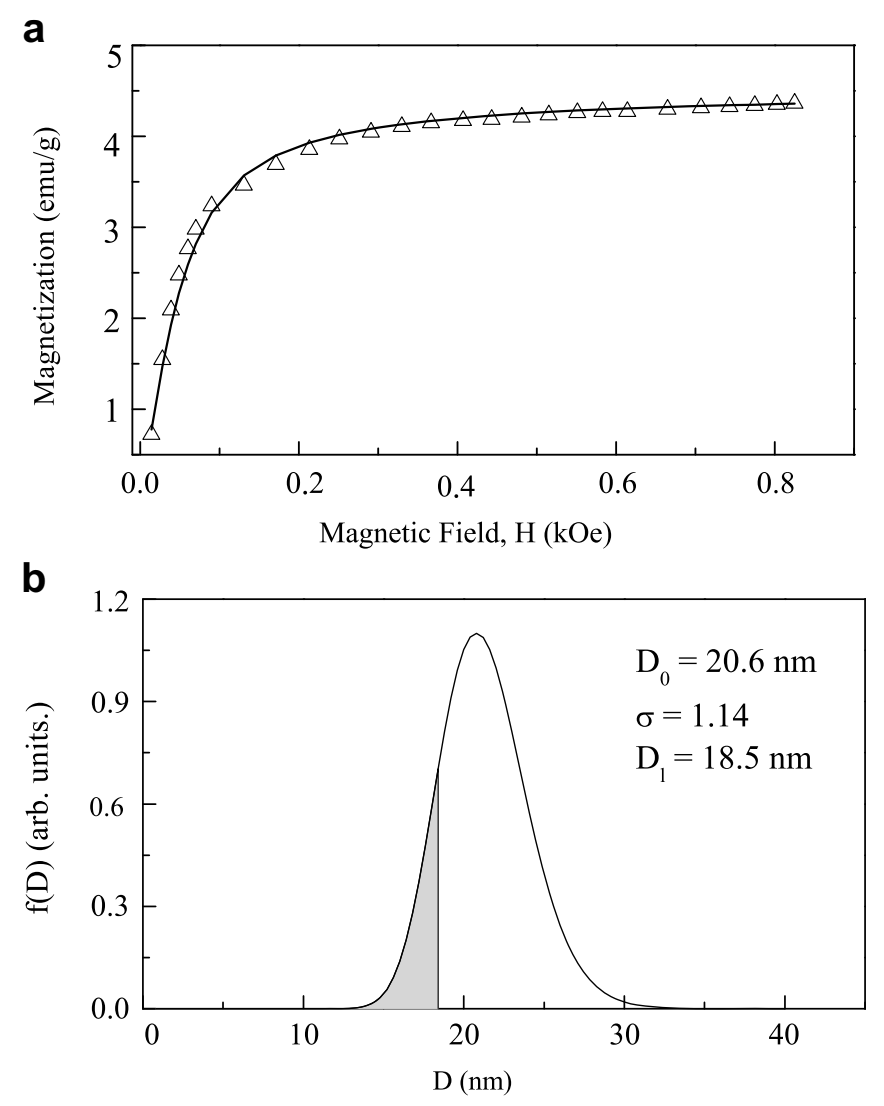

Fig. 6. (a) Experimental (triangles) and fitted (continuous line) curves of magnetization versus applied field for sample A and (b) fitted particle size distribution.

lated for a spherical Fe particle with room temperature anisotropy constant $K=4.8 \times 10^{4} \mathrm{~J} / \mathrm{m}^{3}\left(D_{1}=16 \mathrm{~nm}\right)[15]$.

The ZFC and FC dc magnetic susceptibilities can also be used to extract the parameters of the particle size distribution, as considered by Hansen and Morup [11] following the work done by Wohlfarth [16]. In order to compare the method used by Hansen and Morup to fit the dc magnetic susceptibility curves for singledomain particles with the method presented in this work, we calculate an expression for the ZFC dc mangnetic susceptibility from Eq. (1):

$$
\frac{\chi_{\mathrm{ZFC}}}{25 \mathrm{~K}} \propto \frac{M_{\mathrm{SPM}}^{2}}{3} \int_{0}^{T / T_{B m}} \frac{T_{B m}}{T} f(y) \mathrm{d} y+M_{\mathrm{BL}}^{2} \int_{T / T_{B m}}^{\infty} \frac{T_{B m}}{T} y f(y) \mathrm{d} y,
$$

where $T_{B m} \approx K V_{0} / 25 k_{\mathrm{B}}, K$ is the anisotropy constant, $y=V / V_{0}$ and $V_{0}=\frac{\pi}{6} D_{0}^{3}$.

Fig. 7 shows the fit of the calculated $\chi_{\mathrm{ZFC}}(T)$ curve to the ZFC curve for the sample A. The value obtained for $T_{B m}$ using Eq. (4) is $449 \mathrm{~K}$. This value agrees with that obtained using the method of Hansen and Morup (not shown in the figure), $T_{B m}=447 \mathrm{~K}$. The values of $\sigma$ and $D_{0}$ also agree exactly with those obtained from Fig. 6(b). The particle size distribution obtained from the fit of the magnetization and dc susceptibility data are very close showing a good agreement of the proposed phenomenological model with the experimental data. Moreover, the above results show that the method considered here for fitting $\chi_{\mathrm{ZFC}}(T)$ can be applied to systems composed of single-domain particles as is the case for $\mathrm{Fe}_{10} \mathrm{Ag}_{90}$ granular alloys.

Table 1 shows the values of the parameters $\sigma, M_{\mathrm{SPM}}, M_{\mathrm{BL}}$ and $D_{\mathrm{m}}$ obtained from the fitting, and of $H_{\mathrm{c}}$ and $D_{\mathrm{m}}$ determined from X-ray diffraction of the all samples. We can see a clear correlation between the experimental values and the fitting parameters. For the 


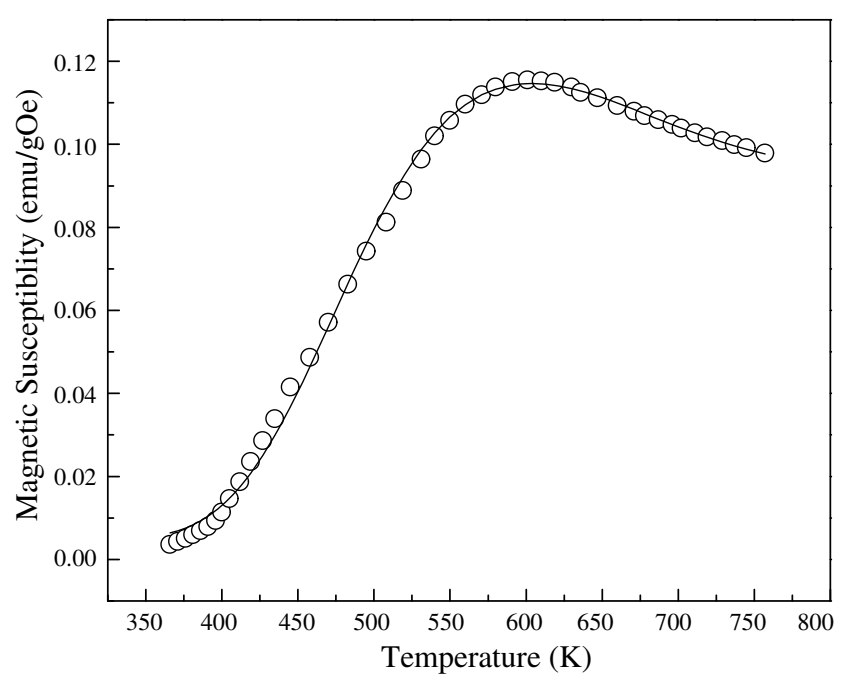

Fig. 7. Experimental (circles) and fitted (continuous lines) ZFC dc magnetic susceptibility versus temperature for sample A and $H=100$ Oe.

Table 1

Coercive field $\left(H_{\mathrm{c}}\right)$, particle distribution width $(\sigma)$, saturation magnetization of SPM $\left(M_{\mathrm{SPM}}\right)$ and $\mathrm{BL}\left(M_{\mathrm{BL}}\right)$ particles, Fe mean particle diameter obtained from X-ray diffraction $\left(D_{\mathrm{RX}}\right)$ and particle size distribution $\left(D_{\mathrm{m}}\right)$ for samples $\mathrm{A}, \mathrm{B}, \mathrm{C}, \mathrm{D}$, and $\mathrm{E}$.

\begin{tabular}{lllllll}
\hline & $H_{\mathrm{c}}(\mathrm{Oe})$ & $\sigma$ & $M_{\mathrm{SPM}}(\mathrm{emu} / \mathrm{g})$ & $M_{\mathrm{BL}}(\mathrm{emu} / \mathrm{g})$ & $D_{\mathrm{RX}}(\mathrm{nm})$ & $D_{\mathrm{m}}(\mathrm{nm})$ \\
& & (adimensional) & & & & \\
\hline A & $354 \pm 4$ & $1.14 \pm 0.02$ & $7.01 \pm 0.10$ & $1.37 \pm 0.02$ & $24.5 \pm 0.8$ & $21.3 \pm 1.1$ \\
$\mathrm{~B}$ & $397 \pm 5$ & $1.36 \pm 0.04$ & $6.00 \pm 0.08$ & $1.70 \pm 0.04$ & $28.0 \pm 1.1$ & $25.8 \pm 1.3$ \\
C & $434 \pm 5$ & $1.67 \pm 0.05$ & $5.15 \pm 0.07$ & $1.59 \pm 0.03$ & $28.6 \pm 1.1$ & $26.3 \pm 1.3$ \\
$\mathrm{D}$ & $403 \pm 4$ & $1.40 \pm 0.03$ & $5.80 \pm 0.08$ & $2.23 \pm 0.04$ & $26.2 \pm 0.8$ & $23.0 \pm 1.2$ \\
E & $376 \pm 4$ & $1.24 \pm 0.03$ & $7.25 \pm 0.10$ & $2.45 \pm 0.05$ & $25.3 \pm 0.7$ & $22.5 \pm 1.1$ \\
\hline
\end{tabular}

sample A we obtain the narrower distribution width $\sigma=1.14$, a high value of the SPM magnetization $M_{\mathrm{SPM}}=7 \mathrm{emu} / \mathrm{g}$, and, as expected, a smaller coercive field $H_{\mathrm{c}}=354$ Oe. However, in the sample $C$, a higher value for $\sigma=1.67$ and a stronger contribution from BL particles to the overall magnetization are obtained. This result is in perfect agreement with the high measured value for the coercivity, $H_{\mathrm{c}}=434 \mathrm{Oe}$. Thus, since the $H_{\mathrm{c}}$ value depends directly on the number of blocked particles in the distribution, the coercive field has a strong dependence on the distribution width. The results of the saturation magnetization of the BL particles obtained from the fits are also in agreement with the values for the remanent magnetization $M_{r}$, considering Stoner-Wohlfarth particle assemblies [17]. Another confirmation of the efficiency of this method is found from the comparison between the mean particle diameter calculated using Eq. 3 and the diameter obtained from X-ray diffraction. The calculated values of $D_{\mathrm{m}}$ vary in the same way as those measured by X-ray dif- fraction. Note that, although the nitric acid concentration considerably influences the particle size distribution, it does not alter $D_{\mathrm{m}}$ significantly. One only sees a small increase in $D_{\mathrm{m}}$ as the acid concentration is increased up to $0.75 \mathrm{ml}$, followed by a gradual decrease for higher concentrations.

\section{Conclusion}

Samples of $\mathrm{Fe}_{10} \mathrm{Ag}_{90}$ granular alloys were produced in various concentrations of nitric acid. This procedure allowed us to vary the diameter of the particles in a controlled way. Magnetic measurements showed the presence of both SPM and BL particles in the particle size distribution. Assuming a single-domain particle size distribution consisting of two components, one SPM and other $\mathrm{BL}$, a method for fitting the magnetization curves to a size distribution was proposed. Although the model ignores dipolar interactions and surface effects, the mean diameters of iron particles calculated using this method agree well with those obtained from $\mathrm{X}$-ray diffraction. The results show that there is a strong correlation between $H_{\mathrm{c}}$ and $\sigma$.

\section{Acknowledgement}

This work was partially supported by the Brazilian agencies CNPq, FINEP, CAPES and FACEPE.

\section{References}

[1] A.E. Berkowitz, J.R. Mitchell, M.J. Carey, A.P. Young, S. Zhang, F.E. Spada, F.T. Parker, A. Hutten, G. Thomas, Phys. Rev. Lett. 68 (1992) 3745

[2] J.Q. Xiao, J.S. Jiang, C.L. Chien, Phys. Rev. Lett. 68 (1992) 3749.

[3] J.M. Soares, J.H. de Araújo, F.A.O. Cabral, T. Dumelow, F.L.A. Machado, A.E.P. de Araújo, Appl. Phys. Lett. 80 (2002) 2532.

[4] B. Abeles, in: R. Wolfe (Ed.), Applied Solid State Science: Advances in Materials and Device Research, Academic Press, New York, 1976, p. 1.

[5] A. Chartterjee, A. Datta, Anit. K. Giri, D. Das, D. Chakravorty, J. Appl. Phys. 72 (1992) 3832.

[6] Jian-Ping Wang, He-Lie Luo, Nai-Fei Gao, Yuan-Yuan Liu, J. Mater. Sci. 31 (1996) 727.

[7] J.A. Gómez, S.K. Xia, E.C. Passamani, B. Giordanengo, E.M. Baggio-Saitovitch, J. Magn. Magn. Mater. 223 (2001) 112.

[8] A.D.C. Viegas, J. Geshev, L.S. Dorneles, J.E. Schmidt, M. Knobel, J. Appl. Phys. 82 (1997) 3047

[9] R. Sappey, E. Vincent, N. Hadacek, F. Chaput, J.P. Boilot, D. Zins, Phys. Rev. B 56 (1997) 14551.

[10] F. Luis, J.M. Torres, L.M. García, J. Bartolomé, J. Stankiewicz, F. Petroff, F. Fettar, J.-L. Maurice, A. Vaurès, Phys. Rev. B 65 (2002) 094409.

[11] M.F. Hansen, S. Morup, J. Magn. Magn. Mater. 203 (1999) 214.

[12] J.C. Denardin, A.L. Brandl, M. Knobel, P. Panissod, A.B. Pakhomov, H. Liu, X.X Zhang, Phys. Rev. B 65 (2002) 064422.

[13] J.M. Soares, J.H. de Araújo, F.A.O. Cabral, J.A.P. da Costa, J.M. Sasaki, Mater. Res. 7 (2004) 513.

[14] P.R. Bevington, Data Reduction and Errror Analysis for the Physical Sciences, McGraw-Hill, New York, 1969. pp. 204-242.

[15] B.D. Cullity, Introduction to Magnetic Materials, Addison-Wesley, Reading, MA, 1972. p. 413.

[16] E.P. Wohlfarth, Phys. Lett. A 70 (1979) 489.

[17] G. Bertotti, Hysteresis in Magnetism: For Physicists, Materials Scientists, and Engineers, Academic Press, Hardback, 1998. 